\title{
Comparative Analysis of Sensory Rhodopsin II Structures in Complex with a Transducer and without It
}

\author{
A. B. Reshetnyak ${ }^{a, c}$, V. I. Borshchevskiy ${ }^{a, c}$, J. Klare ${ }^{b}$, E. S. Moiseeva ${ }^{a, c}$, \\ M. Engelhardt ${ }^{b}$, G. Buldt ${ }^{e}$, and V. I. Gordeliy ${ }^{a, c, d, e}$ \\ a Institute of Structural Biology, Grenoble, 38000 France \\ ${ }^{b}$ Max-Planck Institute of Molecular Physiology, Otto-Hahn-Strasse 11, D-44227 Dortmund, Germany \\ ${ }^{c}$ Physico-Technical Institute, Center of Biophysics and Physico-Chemistry of Permolecular Structures, \\ Institutskii per. 9, Dolgoprudnyi, Moscow oblast, 141700 Russia \\ ${ }^{d}$ Frank Laboratory of Neutron Physics, Joint Institute for Nuclear Research, Dubna, Moscow oblast, 141980 Russia \\ ${ }^{e}$ Institute for Neurobiology and Biophysics 2, Forschungszentrum Jülich, 52425 Jülich, Germany \\ e-mail: g.valentin@fz-juelich.de \\ Received March 12, 2008
}

\begin{abstract}
Sensory rhodopsin II (NpSRII) is a heptahelical transmembrane protein containing the retinal chromophore. In complex with another membrane protein, i.e., NpHtrII transducer, NpSRII transmits a signal into a cell, initiating negative phototaxis of Natronobacterium pharaonis. In the absence of a transducer, rhodopsin II can work as a proton pump similar to bacteriorhodopsin. The main objective of this study is to comparatively analyze receptor structures in complex with the transducer and without it to understand the mechanism of the protein switching function. Diffraction data for sensory rhodopsin II crystals grown in the lipidic cubic phase were obtained at the synchrotron X-ray source. In this paper, we present a new NpSRII structure with a resolution of $2.1 \AA$ and the results of a comparative analysis of the obtained NpSRII structure with our previously published data on the NpSRII/NpHtrII complex structure and with two NpSRII structures without a transducer, previously published in the literature.
\end{abstract}

DOI: $10.1134 / \mathrm{S} 1027451008060128$

\section{INTRODUCTION}

Archaebacterial rhodopsins are photoactive transmembrane heptahelical proteins containing the retinal chromophore bound with lysine in their alpha helix G. The common feature and key stage of activation of these proteins is retinal isomerization under exposure to light. Nevertheless, functions of archaebacterial rhodopsins are different: bacteriorhodopsin (BR) and halorhodopsin (HR) are proton and chlorine ion pumps $[1,2]$, respectively, whereas sensory rhodopsins I and II are receptors of positive and negative phototaxis, which control cell motion as the light intensity and wavelength vary $[3,4]$. Signals are transmitted into the cell by transducer proteins I and II bound with sensory rhodopsin. Activation of transducers initiates a cascade of phosphorylation reactions in the cell, which control the work of flagellar motors of bacteria [5]. However, sensory rhodopsins free of transducer molecules play the role of proton pumps [4]. This, along with a high homology of proteins, allows the assumption that the proton transfer method characteristic of bacteriorhodopsin also works in sensory rhodopsin. The switching mechanism of the function of sensory rhodopsins I and II from proton transfer to signal transmission to the transducer has not yet been known. The study and comparison of the structures of the ground and intermediate states of the photocycle of NpSRII and NpSRII/NpHtrII complex can help to answer the question about the molecular mechanism of a qualitative change in the protein function. Previously, structures of the ground state of sensory rhodopsin II were obtained at a resolution of 2.1 and $2.4 \AA[6,7]$. Moreover, structures of the ground and intermediate states of NpSRII in complex with a transducer were previously obtained in our laboratory $[8,9]$. However, the structures have not yet been comparatively analyzed in detail. Currently, we have solved the NpSRII structure without a transducer, crystallized under the same conditions as NpSRII/NpHtrII complex crystals, which allows for direct comparison of structures.

\section{MATERIALS AND METHODS}

\section{NpSRII Separation and Crystallization}

The NpSRII gene cloned in pET27bmod with seven histidines at the $C$-terminal end were expressed in the BL21(DE3) strain; then the protein was separated and purified as described previously $[10,11]$. Sensory rhodopsin II was reconstructed into membranes of polar lipids of purple membranes of Natronobacterium pharaonis, removing imidazole using diethylaminoethyl chromatography. The reconstructed protein was fil- 
Crystallographic data

\begin{tabular}{|c|c|c|c|}
\hline Data scaling and integration & New model & $1 \mathrm{H} 68$ & $1 \mathrm{H} 2 \mathrm{~S}$ \\
\hline Wavelength, $\AA$ & 0.934 & 0.933 & 0.934 \\
\hline Resolution, $\AA$ & 2.1 & 2.1 & 1.95 \\
\hline \multirow[t]{2}{*}{ Cell sizes, $\AA$} & 86.4129 .151 & 84.8128 .750 .7 & 124.346 .9653 .84 \\
\hline & 909090 & 909090 & 909090 \\
\hline Symmetry & $C 222_{1}$ & $C 222_{1}$ & $P 2{ }_{1}{ }_{1} 2$ \\
\hline Mosaicity & 0.480 .570 .54 & 1.7 & - \\
\hline Number of reflections & 62751 & - & - \\
\hline Number of unique reflections & 16753 & - & - \\
\hline$R_{\text {merge }}^{1}, \%$ (at the outer shell) ${ }^{2}$ & $8.3(42.6)$ & $7.2(42.5)$ & $6.2(39.4)$ \\
\hline$I / \sigma(I)$ (at the outer shell) ${ }^{2}$ & $12.5(3.4)$ & $8.4(1.8)$ & 5.5 \\
\hline Completeness (at the 2 outer shell) ${ }^{2}$ & $98.5(96.2)$ & 96.1 & $94.7(77.3)$ \\
\hline Wilson $B$-factor, $\AA^{2}$ & 22.8 & 23.7 & 32.4 \\
\hline \multicolumn{4}{|l|}{ Model construction and refinement } \\
\hline$R$-factor ${ }^{3}, \%$ (at the outer shell) ${ }^{2}$ & $19.6(20.7)$ & $23.7(27.3)$ & 22.8 \\
\hline$R_{\text {free }}^{4}, \%(\text { at the outer shell })^{2}$ & $24.2(23.6)$ & $25.6(29.5)$ & 25.8 \\
\hline $\begin{array}{l}\text { Root-men-square error in bond lengths, } \AA \text {, } \\
\text { and angles }\end{array}$ & $0.019 / 1.67$ & $0.006 / 1.00$ & $0.009 / 1.07$ \\
\hline
\end{tabular}

tered, precipitated by centrifuging at $100000 \mathrm{~g}$, and resolubilized in a buffer containing $2 \%$ of $n$-octyl-b-Dglucopyranoside (mixing for $16 \mathrm{~h}$ at $T=4^{\circ} \mathrm{C}$ in dark). Resolubilized sensory rhodopsin II in $25 \mathrm{mM} \mathrm{Na} / \mathrm{K}-\mathrm{Pi}$ buffer $(\mathrm{pH}=5.1$ ) containing $150 \mathrm{mM} \mathrm{NaCl}$ and $0.8 \%$ $n$-octyl-b-D-glucopyranoside was crystallized at $22^{\circ} \mathrm{C}$ from the lipidic cubic phase formed by monoolein [12]. As precipitant, $\mathrm{Na} / \mathrm{K}-\mathrm{Pi}(\mathrm{pH}=5.15)$ was used.

\section{X-Ray Diffraction Data}

X-ray diffraction data were obtained at the ID14-EH1 protein crystallography station in the European Synchrotron Radiation Facility (ESRF, Grenoble, France) at a wavelength of $0.934 \AA$ using the ADSC Q4R CCD detector (Poway, United States). For the diffraction experiments, crystals in a cryoloop (Hampton Research, United States) were rapidly frozen in a nitrogen cryojet to $100 \mathrm{~K}$. After that, a complete set of data were measured, which was obtained in this symmetry by the crystal rotation over $90^{\circ}$. The oscillation angle for each diffraction pattern was $1^{\circ}$, the exposure time was $10 \mathrm{~s}$. The data obtained were processed using the MOSFLM and SCALA programs from the CCP4 Suite crystallographic package [13]. The data resolution is $2.1 \AA$, the crystal symmetry is $C 222_{1}$. Initial phases were obtained by molecular replacement by the polyalanine chain (according to the model published in [7]) using the MOLREP program (CCP4). The model was automatically constructed and refined using the ARP/WARP and REFMAC 5 programs (CCP4), respectively. The electron density map was studied using the Coot program [14].

\section{RESULTS AND DISCUSSION}

Protein crystals of sensory rhodopsin were grown in the cubic phase of monoolein, diffraction patterns were measured at the ESRF and processed using the phases (as initial ones) obtained by replacing amino acids of the sensory rhodopsin model (constructed in [7], pdbcode of the model 1H68) by alanines. The model consists of one protein molecule, i.e., sensory rhodopsin, and 35 water molecules. Moreover, oblong cylindrical areas corresponding to the lipidic bilayer, which were interpreted as aliphatic chains of lipidic molecules, were detected in the electron density maps.

Crystallographic characteristics of the data obtained and statistics for the constructed model are listed in the table. Also presented is a comparison of the data obtained in this study with the previously published structures of sensory rhodopsin and its complex with a transducer (pdb-code of the model $1 \mathrm{H} 2 \mathrm{~S}$ ). As seen in the table, some important crystallographic parameters characterizing the diffraction data and model quality 


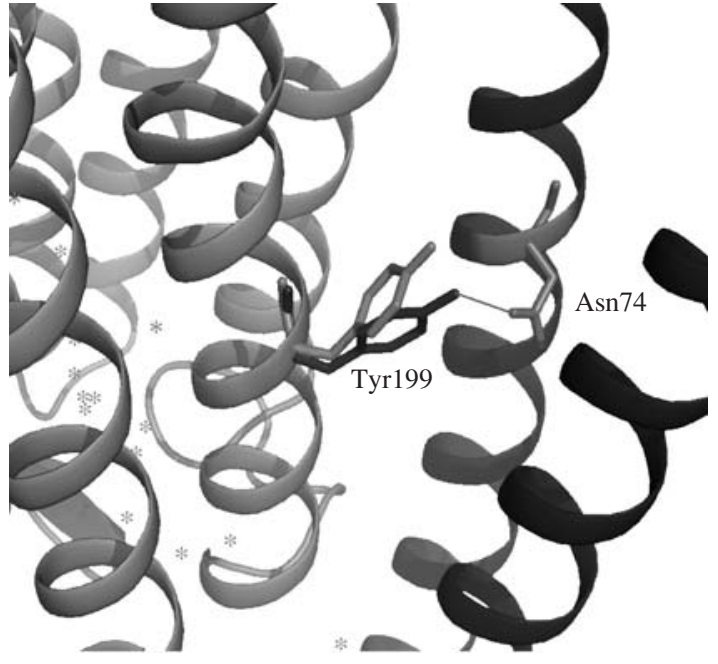

Fig. 1. Change in the Tyr199 conformation during the interaction with a transducer: the transducer and Tyr199 conformation in complex are shown by a dark color; the single receptor and the Tyr199 conformation in it are shown by a bright color.

(the mosaicity, crystallographic $R$-factor of the model, and Wilson $B$-factor) were improved in comparison to those published previously.

\section{Comparison of the Structures of the Bacterial Rhodopsin Family}

In general, the sensory rhodopsin structure is similar to the bacteriorhodopsin and halorhodopsin structure, so that only small differences are responsible for the difference in functions and characteristics of these proteins. In particular, the difference in the active center structure is responsible for the blue shift of sensory rhodopsin absorption ( $488 \mathrm{~nm}$ ) in comparison with bacteriorhodopsin $(570 \mathrm{~nm})$. As noted previously $[6,15]$, this difference in absorption wavelengths is explained as follows. First, the retinal polycarbon chain of sensory rhodopsin is bent less due to the difference in amino acid residues in the retinal-binding pocket. Second, $\operatorname{Arg} 72$ (Arg82 in BR) is directed toward the extracellular protein portion, whereas its analogue is directed toward the Schiff base (SB). This causes an increase in the distance between Arg72 and Asp75, which results in a decrease in the Asp75 acidity and enhances the SB-counterion interaction [16]. Third, hydroxyl residues Ser141 and Thr142 surrounded by the retinal betaionone ring are replaced by neutral Gly130 and Ala131 in sensory rhodopsin [17]. Another important difference is the replacement of the Asp96 proton donor in bacteriorhodopsin by Phe86 in sensory rhodopsin, which results in a slowed down decay of the intermediate M-state [18].

\section{Comparison of the Structures of Sensory Rhodopsin and Its Complex with a Transducer}

The main objective for the construction of the new model of sensory rhodopsin was to clarify structural changes in sensory rhodopsin, caused by transducer molecule attachment. This problem is of particular interest, since the transducer molecule attachment causes a change in the sensory rhodopsin function from signal transmission to proton pumping, and the mechanism of such switching remains unknown. It was necessary to obtain crystals under conditions close to those in which crystals of receptors in complex with a transducer were grown, in order to correctly compare the structures and to attempt to find the differences responsible for receptor function switching.

The sensory rhodopsin-transducer protein bond is mainly caused by Van der Waals interactions; hydrogen bonds exist only between Tyr199 and Asn74 (TM1) and between Thr189 and Glu43 (TM1), Ser 62 (TM2) [6, 8]. A comparison of NpSRII and NpSRII/NpHtrII models shows that the transducer molecule attachment does not lead to significant structural changes. A noticeable difference in the structure consists in the turn of Tyr199 in the complex structure toward transducer helices with hydrogen bond formation between Tyr199 and Asn74 of the transducer transmembrane helix TM2 (Fig. 1). An analysis of the structures shows a sharp jump of atomic temperature factors at Tyr199 of sensory rhodopsin in the complex model in comparison with our model and the 1H68 model (Fig. 2). Probably, this is because Tyr199 in the NpSRII crystal forms hydrogen bonds with protein of a neighboring cell, and this lowers its free energy. It is noteworthy that three of six incorporated aliphatic lipid chains are in the exact region of the receptor-transducer contact, more precisely near helix $G$ (Fig. 3). Presumably, this causes protein stabilization after transducer detachment.

Another difference between the two models is the larger number of water molecules in the complex model, i.e., 39 in the complex structure and 35 in the sensor rhodopsin model. The water molecules in the channel inside the protein, which are involved in proton transfer, are identical, except for molecule no. 19 (numbering according to the pdb model of the $1 \mathrm{H} 2 \mathrm{~S}$ complex) which presents itself only in the complex model. This molecule is at the helix base, in the region of proton release and has a high $B$-factor. Therefore, its absence in the model under consideration is probably caused by a poor resolution in comparison with the data on the complex, which does not allow observation of strongly mobile structural elements. The difference in the position and number of water molecules in hydrophilic protein regions is caused by the difference in molecular packing in crystals; in the case of the NpSRII/NpHtrII complex, packing is denser, which probably allows for the fixing of a larger number of water molecules. For example, positions 27, 28, and 1 ( $Y$-chains) of water molecules are caused by the pres- 


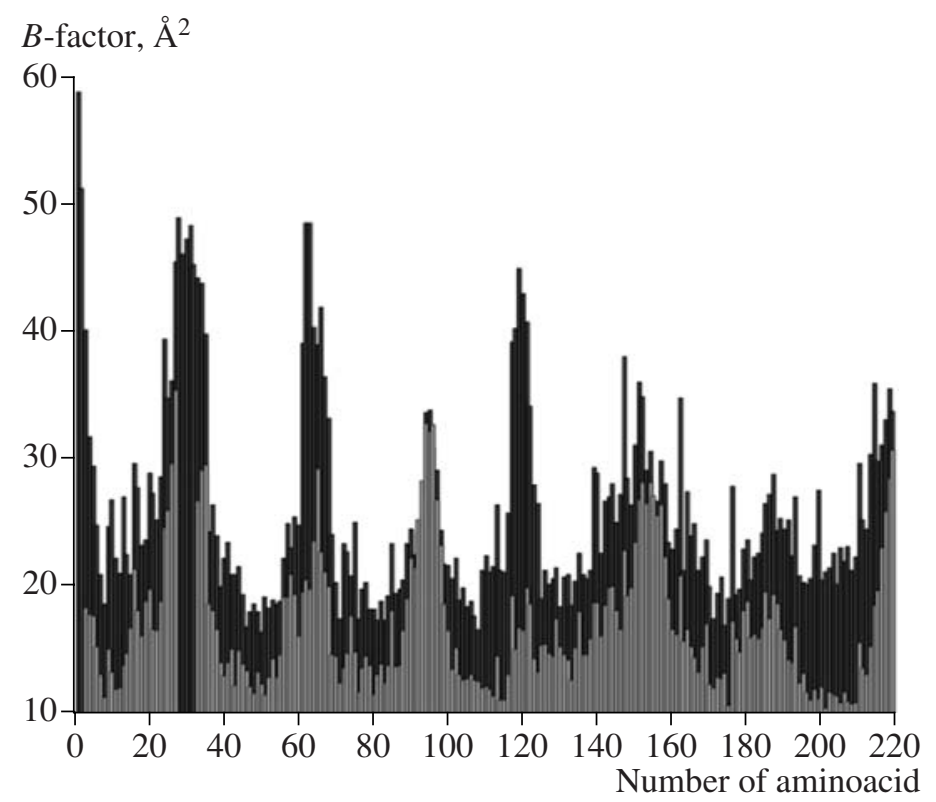

Fig. 2. Statistics of $B$-factors in the $1 \mathrm{H} 2 \mathrm{~S}$-complex model (black) and the new sensory rhodopsin model (gray).

ence of a transducer. The high mobility of the $C$-terminal end of protein and the $A B$-loop of sensory rhodopsin did not allow completion of the protein model in these regions. In the complex model, these regions are completed; there are water molecules 1, 2, 35, 36, 37, 38, and 39 bound with $C$-terminal end amino acids and with each other by hydrogen bonds (Fig. 4). $B$-factors of water molecules in hydrophilic regions indicate their high mobility. Water molecules 4, 16, and 24 are bound by hydrogen bonds with protein molecules in adjacent cells; however, their $B$-factors are large enough. Thus, we can conclude that the difference in water molecule positions is insignificant for protein functioning.

\section{Comparison of the Sensory Rhodopsin Model Developed in our Laboratory with the 1 H68 Model (Royant et al.)}

Among the two published structures of sensory rhodopsin $[6,7]$, the $1 \mathrm{H} 68$ model features a better resolution; therefore, namely it was chosen for a comparative analysis with the new structure of sensory rhodopsin. In general, the models are identical; however, there are also differences. The $1 \mathrm{H} 68$ model includes a chlorine ion; however, we did not detect an electron density higher than that of the water molecule at this site; hence, there were no reasons to incorporate the chlorine atom into the model. The water molecule incorporated instead of chlorine, forms hydrogen bonds with the environment. The presence of chlorine at the 1H68 model can be associated with different crystallization conditions: in the case of the 1H68 model, crystals were grown at a high $\mathrm{NaCl}$ concentration $(1.5 \mathrm{M})$. Asp193, in both our model of sensory rhodopsin and in the complex model is directed into the water channel, in con- trast to the 1H68 model which also includes an additional water molecule forming a hydrogen bond with Asp193 and hypothetically involved in the proton transfer process (Fig. 5). This difference can be explained by the electrostatic interaction of Asp193 with the chlorine ion, resulting in a site for a new water molecule. An analysis of interatomic distance also showed a shift of $\sim 0.5 \AA$ of the $F$-helix in the intracellular protein region in the model by Royant et al. in comparison with our structure of sensory rhodopsin (Fig. 6). This is a paral-

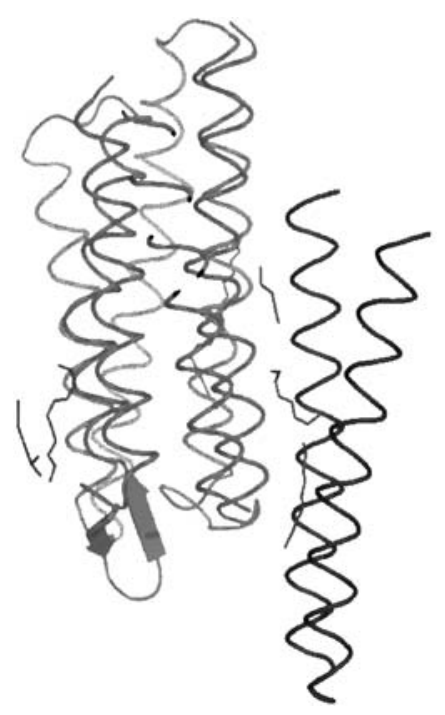

Fig. 3. Lipids incorporated into the sensory rhodopsin model: sensory rhodopsin (new model) is shown by a brighter color, the transducer arrangement with respect to the receptor and lipids (according to the $1 \mathrm{H} 2 \mathrm{~S}$ complex model) is shown by a dark color, lipid chains are dark gray on receptor sides. 


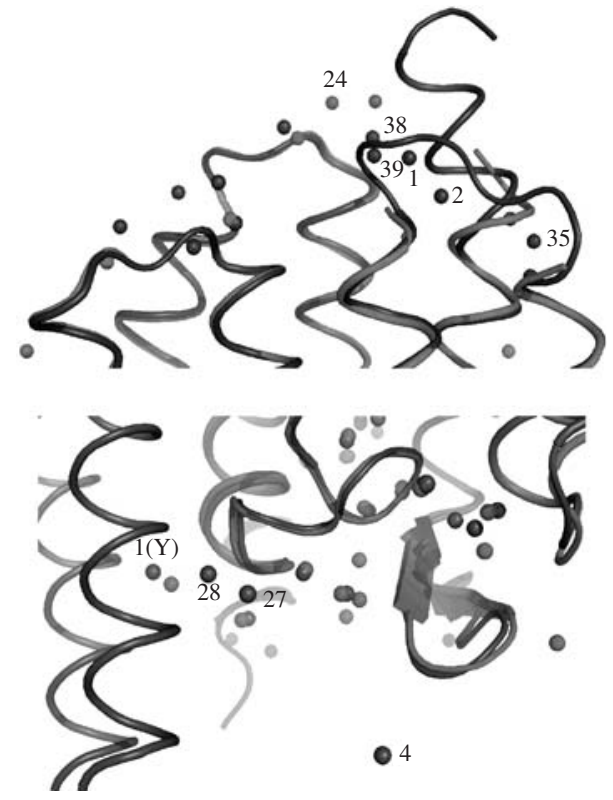

Fig. 4. Differences in water molecule positions in hydrophilic regions between the new model and complex model: the NpSRII model is brighter; the NpSRII/NpHtrII complex model is darker. Numbering is according to the pdb file of the complex.

lel shift; conformations of amino acid residues are almost identical. The hydrogen bond structure is also retained; therefore, it is low probable that this difference has a functional significance. We note that a $0.5-\AA$ shift can also be detected at a crystallographic resolution larger than this value. A resolution of $2.1 \AA$ given in the table corresponds to a minimum distance between atomic planes at which diffraction occurs and characterizes structural details, whereas the atomic position corresponding to peaks of electron planes can be determined with a much better accuracy at high data quality ( $R$-factor, $B$-factor) [21].

In the water channel in the cytoplasmic region, differences in water atomic positions are observed (Fig. 7). For example, water molecules 21,22 , and 29 in the water channel are absent in the 1H68 model, but present in the complex model and new model. These molecules are bound by hydrogen bonds with Arg72, Asp193, Tyr73, and Tyr174, a part of them is directly involved in the protein photocycle. For example, Arg72 is a part of the proton transport chain, and Asp193 shifts together with the $F$-helix in the $M$-state. Water molecule 405 is also bound with Asp193. These water molecules shift with respect to initial positions during the transition to the $M$-state [10]. In the model by Royant et al., water molecules 21, 22, and 29 are absent, and molecule 405 has a changed position, which is probably also caused by different crystallization conditions. In the $1 \mathrm{H} 68$ model in the water channel, water molecule 407 bound by hydrogen bonds with Thr67 and Arg66 is observed at the cytoplasmic region. In the new model and com-

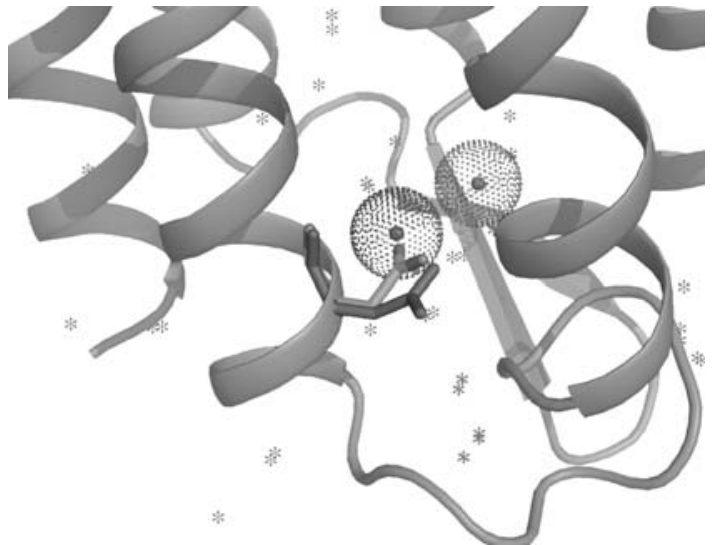

Fig. 5. Change in the Asn 193 conformation: water molecule 405 of the $1 \mathrm{H} 68$ model is right among large spheres, the chlorine ion of the same model is left among them; the Asn 193 conformation of the $1 \mathrm{H} 68$ model is shown by a dark color; the structure of the new model of sensory rhodopsin is shown by a bright color. We can see that Asn 193 is directed into the proton channel in the latter model, which results in that water 405 finds positions in the new model.

plex model, this water molecule was not detected. The double conformation of water molecule 406 existing in the $1 \mathrm{H} 68$ model also was not detected. This molecule plays an important role in the photocycle, since it is a part of the internal network of hydrogen bonds over which proton transport occurs.

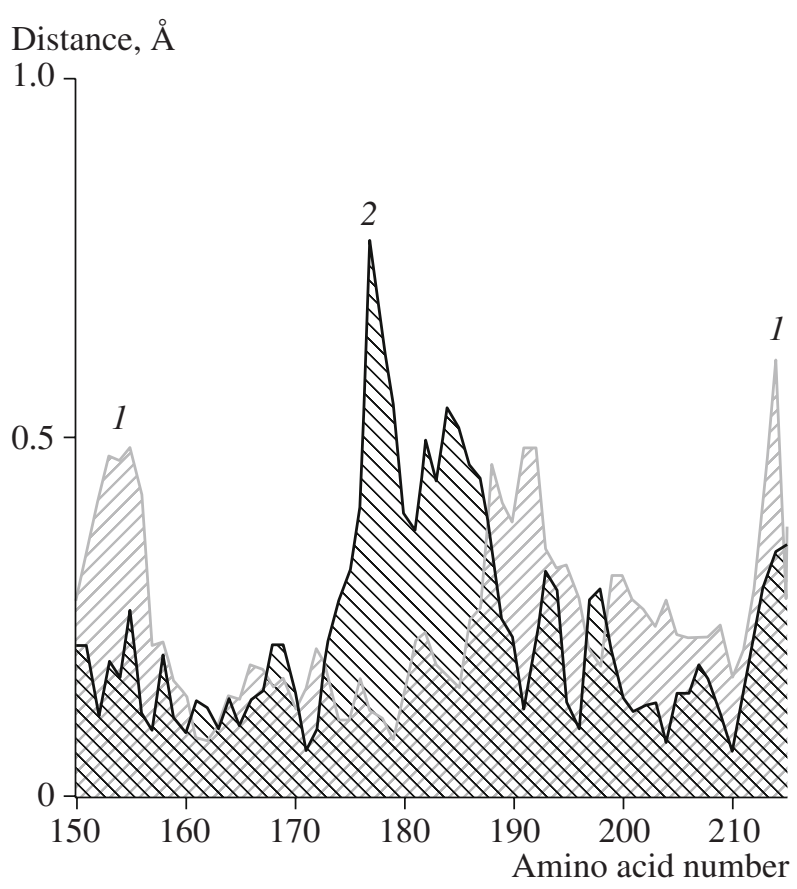

Fig. 6. Root-mean-square distance between atoms of the polypeptide chain between the new model of sensory rhodopsin and the structure of sensory rhodopsin in complex (1), and the new model and 1 H68 (2). The peak in the region of the 170-185th bases corresponds to the $F$-helix shift. 


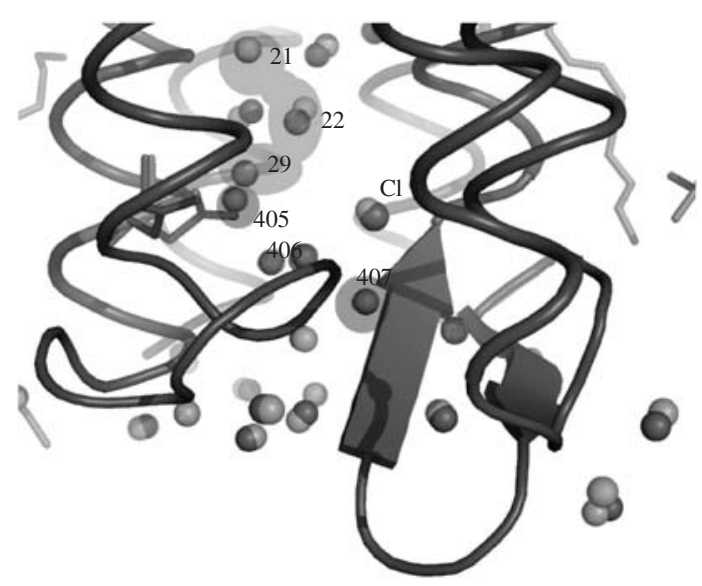

Fig. 7. Difference in water molecule positions in the $1 \mathrm{H} 68$ model and the new model of sensory rhodopsin. The new model is shown by a darker color.

\section{CONCLUSIONS}

The detected differences between sensory rhodopsin models constructed in different laboratories indicate the significant effect of crystallization conditions on the protein structure. Therefore, when comparing the structures of sensory rhodopsin in complex with a transducer and without it, it seems important that crystals of both proteins were grown under similar conditions. However, a comparison of the structures of sensory rhodopsin in complex and without did not show significant differences and has not yet answered the question about the mechanism of switching of sensory rhodopsin functions during transducer attachment. These data suggest that the key to understanding this mechanism is in the generation and analysis of intermediate states of sensory rhodopsin and their comparison with intermediate states sensory rhodopsin in complex with a transducer [10]. First of all, this concerns the $M$-state, since it was shown that it corresponds to the signal state of the receptor $[20,21]$.

\section{REFERENCES}

1. D. Oesterhelt, Curr. Opin. Struct. Biol. 8, 489 (1998).

2. J. K. Lanyi, J. Phys. Chem. 104, 11441 (2000).

3. J. L. Spudich, C.-S. Yang, K.-H. Jung, and E. N. Spudich, Ann. Rev. Cell Dev. Biol. 16, 365 (2000).

4. J. Sasaki and J. L. Spudich, Biochim. Biophys. Acta 1460, 230 (2000).

5. J. L. Spudich, Mol. Microbiology 28, 1051 (1998).

6. H. Luecke, B. Schobert, J. K. Lanyi, et al., Science 293, 1499 (2001).

7. A. Royant, P. Nollert, and K. Edman, Proc. Natl. Acad. Sci. USA 98, 10131 (2001).

8. I. Gordeliy, J. Labahn, and R. Moukhametzianov, Nature 419, 484 (2002).

9. R. Moukhametzianov, J. P. Klare, and R. Efremov, Nature 440, 115 (2006).

10. I. P. Hohenfeld, A. A. Wegener, and M. Engelhard, FEBS Lett. 442, 198 (1999).

11. K. Shimono, M. Iwamoto, M. Sumi, and N. Kamo, FEBS Lett. 420, 54 (1997).

12. M. Caffrey, J. Struct. Biol. 142, 108 (2003).

13. Collab. Computational Project No. 4 (1994); "The CCP4 Suite: Programs for Protein Crystallography," Acta Crystallogr. D 50, 760 (1994).

14. P. Emsley and K. Cowtan, Acta Crystallogr. D 60, 2126 (2004).

15. B. Yan, J. L. Spuditch, and P. J. Mazur, J. Biol. Chem. 270, 29668 (1995).

16. K. Nakanishi, V. Balogh-Nair, and M. A. Gawinowicz, Photochem. Photobiol. 29, 657 (1979).

17. J. L. Spudich, D. A. McCain, and K. Nakanishi, Biophys. J. 49, 479 (1986).

18. M. Iwamoto, K. Shimono, M. Sumi, and N. Kamo, Biophys. Chem. 79, 187 (1999).

19. J. P. Klare, I. Gordeliy, and J. Labahn, FEBS Lett. 564, 219 (2004).

20. B. Yan, T. Takahashi, R. Johnson, and J. L. Spudich, Biochem. 30, 10686 (1991).

21. I. Gordeliy and I. Chernov, Acta Crystallogr. D 53, 377 (1997). 